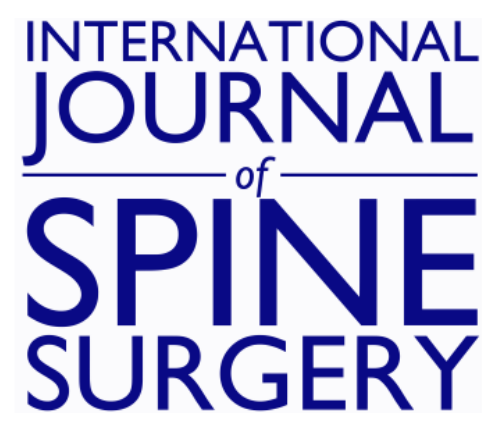

\title{
Changes in Neuroforaminal Height with 2 Level Axial Presacral Lumbar Interbody Fusion at L4-S1
}

Satyajit Marawar, Nathaniel Ordway, Jin Jung and Mike Sun

Int J Spine Surg 2014, 8 ()

doi: https://doi.org/10.14444/1002

http://ijssurgery.com/content/8/2

This information is current as of April 26, 2023.

Email Alerts Receive free email-alerts when new articles cite this article. Sign up at:

http://ijssurgery.com/alerts

The International Journal of Spine Surgery

2397 Waterbury Circle, Suite 1,

Aurora, IL 60504, Phone: +1-630-375-1432 


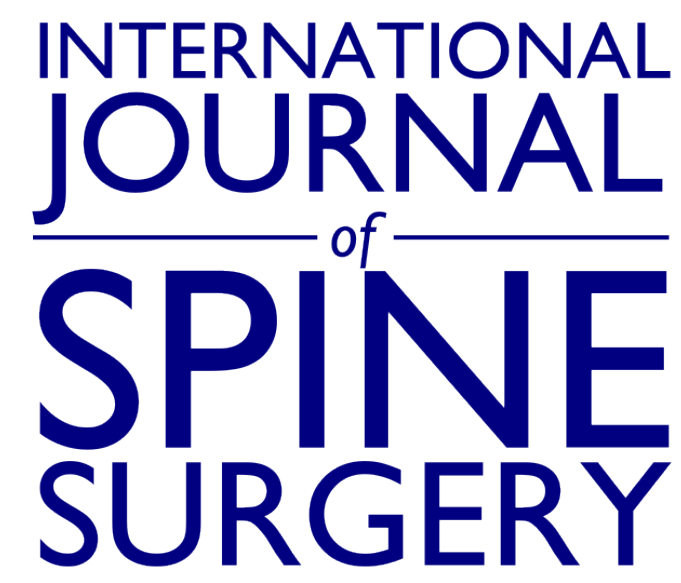

This article generously published free of charge by the International Society for the Advancement of Spine Surgery.

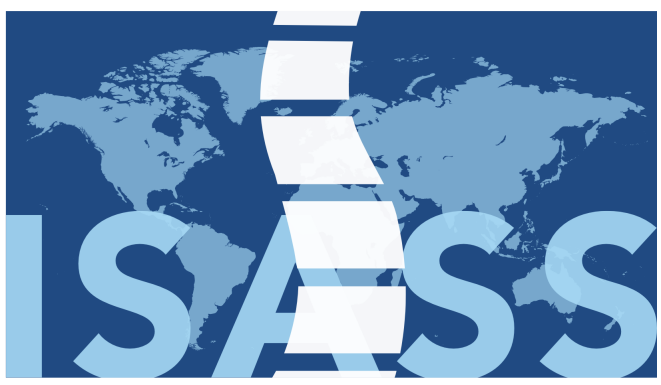

INTERNATIONAL SOCIETY for the ADVANCEMENT of SPINE SURGERY 


\section{Changes in Neuroforaminal Height with 2 Level Axial Presacral Lumbar Interbody Fusion at L4-S1}

Satyajit Marawar, MD, Nathaniel Ordway, MS, Jin Jung, MD, Mike Sun, MD

Orthopedic Surgery, SUNY Upstate Medical University

\section{Abstract}

\section{Background}

The objective was to examine the changes in neuroforaminal height at L4-L5 and L5-S1 after insertion and graduated foraminal distraction using the 2 level transsacral implant in a cadaveric model.

\section{Methods}

Discectomy and transsacral instrumentation was performed in six fresh human cadavers at L4-S1. The neuroforaminal height was measured at L4-L5 and L5-S1 before and after insertion of the implant and then at each stage of manual distraction.

\section{Results}

Mean L4-5 neuroforaminal height increased from $18.2 \pm 3.1 \mathrm{~mm}$ to $20.3 \pm 2.9 \mathrm{~mm}(11 \%)$ on the left and from $18.8 \pm 2.8 \mathrm{~mm}$ to $20.6 \pm 2.3 \mathrm{~mm}(12 \%)$ on the right $(\mathrm{P}<0.05)$. Mean L5-S1 neuroforaminal height increased from $15.7 \pm 3.0 \mathrm{~mm}$ to $18.4 \pm 2.8 \mathrm{~mm}(17 \%)$ on the left and from $15.6 \pm 2.1 \mathrm{~mm}$ to $18.3 \pm 1.8 \mathrm{~mm}(17 \%)$ on the right $(\mathrm{P}<0.05)$. When the neuroforaminal height was plotted against amount of rotation of the screw driver it was found that the neuroforaminal height at L5-S1 increased by $1 \mathrm{~mm}$ on average for every complete revolution of the screw driver. At least 2 full rotations of the screw driver were achieved in all cadavers.

\section{Conclusions}

The transsacral screw construct distracted the disc space and neuroforaminal height in a cadaveric spine model without soft tissue envelope. During the initial process, manual control of disc space distraction predictably correlated with the increase in the neuroforaminal height to a maximum. However, further research is needed to look at variables affecting disc space pliability, implant subsidence, in vivo application, and clinical benefit of this procedure. 


\section{Introduction}

Symptomatic disc degenerative disease with or without instability is commonly seen at L4-L5 and L5-S1 levels. Surgical management of this pathology involves decompression and fusion of the affected levels. Traditional open and newer minimal access techniques have been used to achieve these goals. Minimal access procedures are technically demanding and involve a learning curve. Attempts are being constantly made to develop and perfect new minimal access procedures. Paracoccygeal approach to L5-S1 junction with transsacral instrumentation using AXIALIF implant has been developed in recent years and has shown promising clinical results and reliable biomechanical stability. Biomechanical evaluations of a two level implant for L4-S1 fusion showed encouraging results which have led to clinical application of the two level implant. ${ }^{1}$ Clinical results from two level implants are being evaluated. The transsacral screw is a two piece assembly that consists of an L4-L5 rod and a sacral anchor lagged together by a distraction rod. This transsacral screw is designed to distract the disc spaces to allow space for the bone graft and increase the size of the neuroforamen. After insertion of the implant the L4-L5 disc space is distracted by the thread pitch differential in the L4 and L5 components of the L4-L5 rod. At L5-S1 the disc space is manually distracted by a specialized screw driver that engages the distraction rod. Segmental distraction with the transsacral screw can lead to increases in the neuroforaminal dimensions. This may lead to indirect decompression of the nerve roots which may provide relief from radicular symptoms and avoid direct decompression in well selected cases. However there have been no studies on changes in neuroforaminal dimensions after insertion of the transsacral screw. The purpose of this study was to examine the changes in neuroforaminal height at L4-L5 and L5-S1 after insertion and graduated foraminal distraction using the 2 level transsacral implant in a cadaveric model.

\section{Materials and Methods}

Six fresh-frozen human cadaveric lumbar spine specimens (T12-S1) were dissected (4F/ $2 \mathrm{M}$, age $70.3 \pm 25.7$ years, height $174 \pm 9 \mathrm{~cm}$, weight $85.9 \pm 15.6 \mathrm{~kg}$,). The bone mineral density of each specimen was evaluated using a DEXA scanner (GE Lunar DPX-IQ) and a lumbar spine protocol (T-score: $-0.3 \pm 1.4$ ). Each lumbar spine was cleaned of musculature and neural structures, but the facet joints and all ligamentous structures were kept intact. All the specimens were screened via fluoroscopy in order to rule out any major anatomical abnormality (e.g., severe degeneration, fracture, deformity, dysplasia, pars defects, or congenital anomaly).

Each specimen was warmed to room temperature before the procedure to avoid effects of temperature on the nitinol components of the instrumentation system. Neuroforaminal height measures were initially collected prior to the procedure. A manual caliper (accuracy of $0.05 \mathrm{~mm}$ ) was used to measure the foraminal height. This technique was adopted because this technique has shown a lower interobserver and intraobserver variability ( $\kappa=0.79$ and 0.81 , respectively) compared with measurements performed on CT scans or silicon molds of the foramen ( $\kappa<0.76$ and $\kappa<0.65$, respectively). ${ }^{2}$ All measurements were performed using the same technique. The vertical dimension, or 
foraminal height, is the shortest distance between the inferior cortex of the upper pedicle and the superior cortex of the lower pedicle. At both L4 and L5 the inferior surface of the pedicle exhibits a concave shape and has an oblique orientation in the coronal plane, the medial border being located cranially with respect to the lateral border, which thus represents the most inferior portion of the pedicle. This inferolateral border, however, is usually located laterally to the top of the pedicle below; therefore, it cannot be used as a bony landmark for the measurement of the foraminal height. So at both L4-L5 and L5-S1 the narrowest vertical diameter through which the nerve root has to pass was measured as a vertical line drawn from the top of the lower pedicle to the inferior border of the upper pedicle (Figure 1). Bilateral neuroforaminal heights at both L5-S1 and L4-L5 were measured preoperatively and recorded as baseline measures.

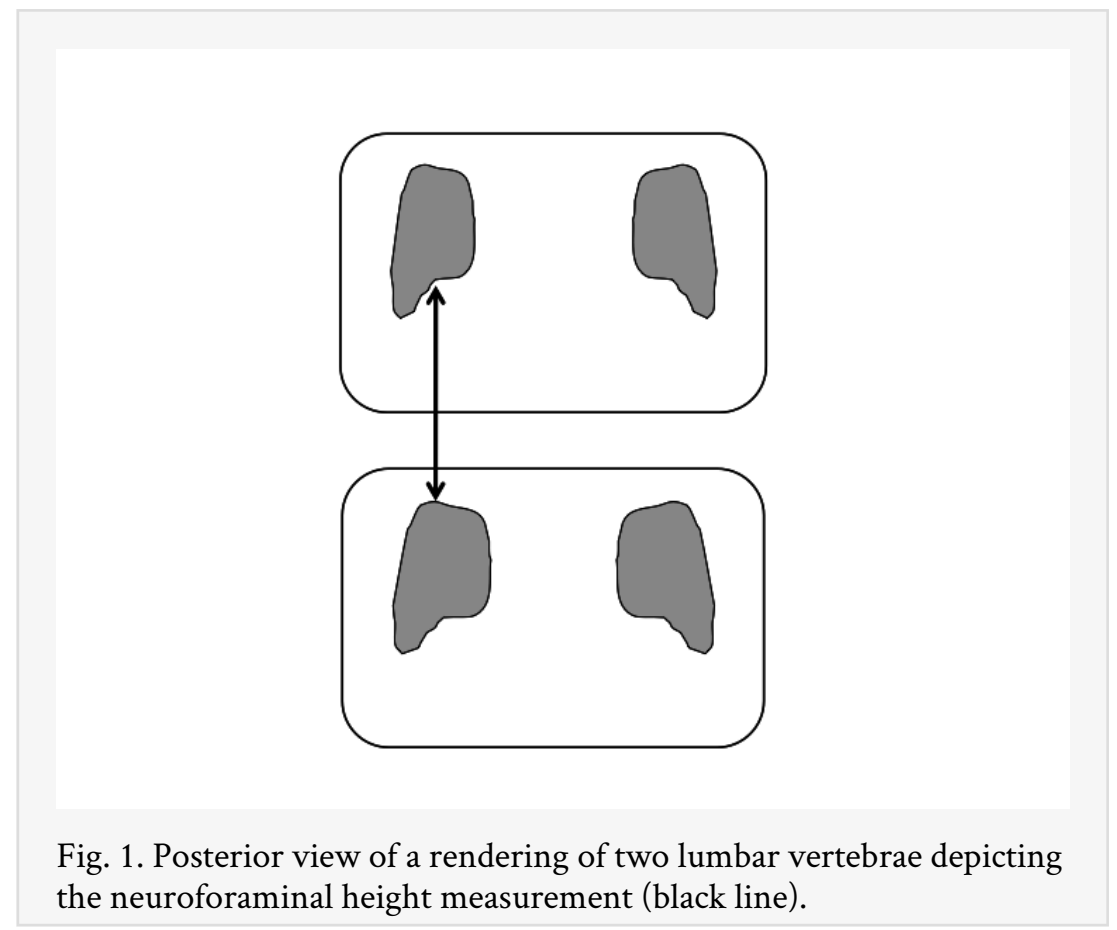

The insertion of the transsacral implant was performed in a standard manner under fluoroscopic control by a spine surgeon trained in performing the procedure. The technique for insertion involved passing in a series of dilators over a guide wire inserted in the L4-S1 disc spaces. A series of reamers are passed over the guide wire to establish a working channel into the disc spaces. A thorough discectomy is performed through the working channel using radial disc debulkers, disc extractors and end plate rasps (Figure 2). 


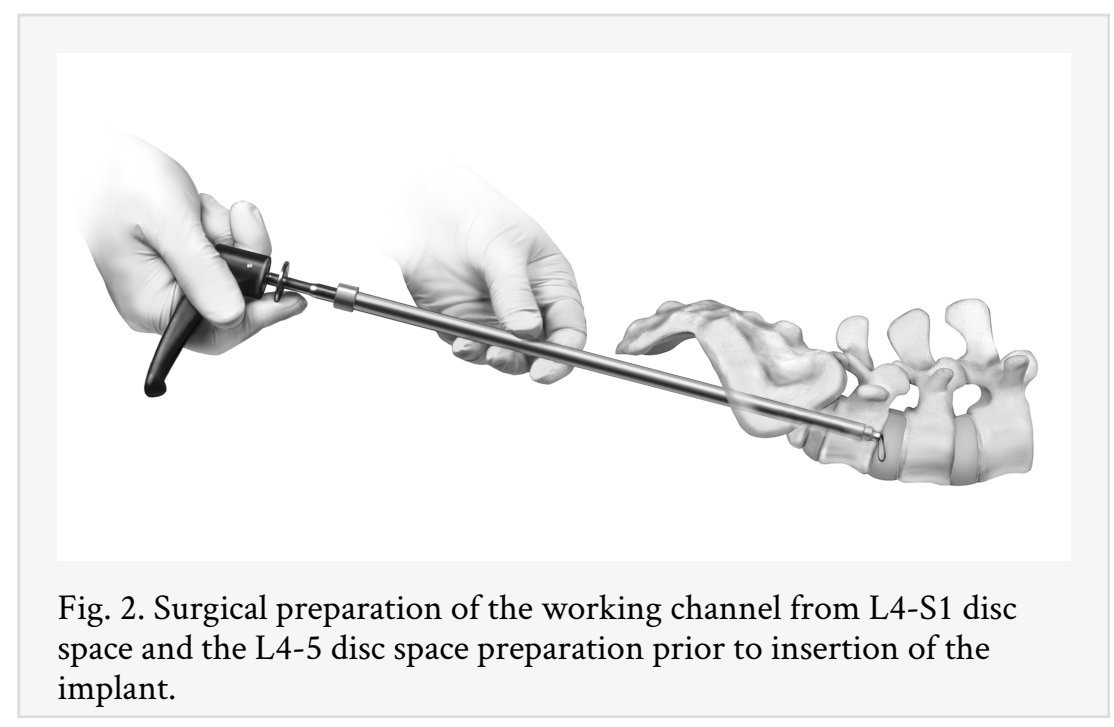

After discectomy the size of the presacral implant (Trans1, Inc., Wilmington, NC, model AxiaLIF $2 \mathrm{~L}+$ ) to be inserted was finalized using $\mathrm{c}$ arm images and standard templates provided by the manufacturer. Bone graft was not inserted into the disc spaces for the cadaveric procedure. The implant was a 2 piece assembly that consisted of the L4-L5 rod and the sacral anchor lagged together by a distraction rod. The L4-L5 disc space is distracted by the pitch differential between the L4 and L5 components of the L4-L5 rod (Figure 3). The L5-S1 disc space is distracted manually by a screw driver that engages the distraction rod that lags the L4-L5 rod with the S1 anchor (Figure 3). At L4-L5 various levels of pitch differential are available and a pitch differential level of 10-12 was used in this study. This is the maximum pitch differential offered with the implant and meant there were 10 threads per inch in the L5 section and 12 threads per inch in the L4 section of the L4-L5 rod. A fixation rod locked the implant after completion of manual distraction (Figure 3 ).

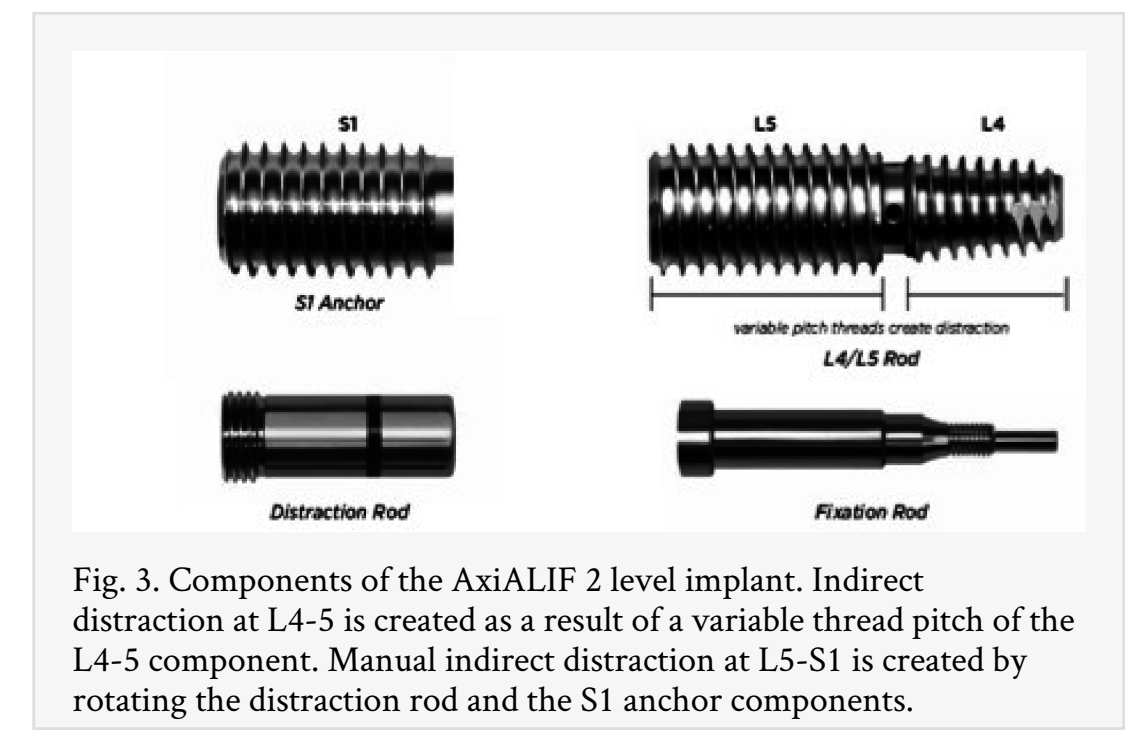

Following confirmation of acceptable implant placement using fluoroscopy, the neuroforaminal height was measured bilaterally at both L4-L5 and L5-S1. Then graduated distraction at L5-S1 was started. The screwdriver for L5-S1distraction was inserted and 
rotated until the L4-L5 rod and S1 anchor were engaged by the distracting mechanism. An increase in resistance to the rotation of the screwdriver signaled the beginning of the distraction of the L5-S1 disc space. Distraction was carried out by rotating the screwdriver through 180 degrees (a half a revolution at a time) and then measuring the neuroforaminal heights of L4-5 and L5-S1. This process was continued till the screwdriver lost resistance or the implant was seen backing out at the sacrum. C arm images were taken at regular intervals to monitor the distraction process and to evaluate the implant for loosening. Figure 4 shows $\mathrm{C}$ arm images before and after distraction at the L5-S1 disc space in one of the cadavers.

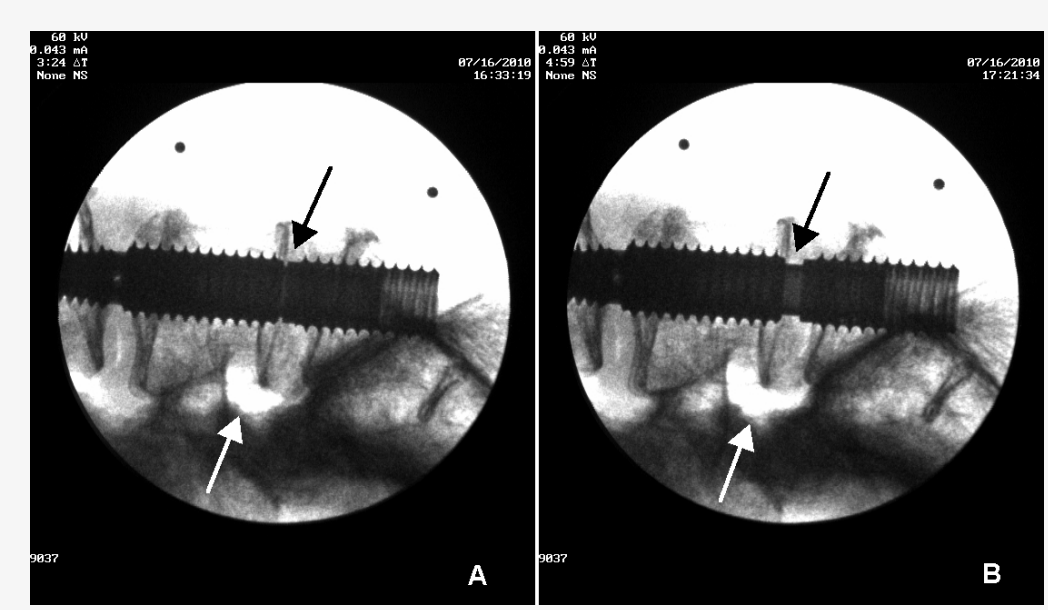

Fig. 4. An example lateral fluoroscopic view of a lumbar specimen with the implant in place A) prior to manual distraction and B) after manual distraction is complete. Black arrows show the change in displacement of the distraction rod and the white arrows show the resulting change in the neuroforamen.

The neuroforaminal heights at L4-5 were statistically analyzed and student's t-test comparisons were made to examine differences between baseline and post-implant measurements bilaterally as well as differences in symmetry between the left and right neuroforamen. The neuroforaminal heights at L5-S1 were averaged and plotted for each half revolution until maximal distraction was reached. Student's t-test comparisons were made to examine differences between baseline and maximal distraction measurements bilaterally as well as differences in symmetry between the left and right neuroforamen. The slopes of the neuroforaminal height vs distraction curves were analyzed using regression analysis. A significance level of $\alpha=0.05$ was used for all statistical analyses.

\section{Results}

Mean L4-5 neuroforaminal height increased from $18.2 \pm 3.1 \mathrm{~mm}$ to $20.3 \pm 2.9 \mathrm{~mm}$ on the left and from $18.8 \pm 2.8 \mathrm{~mm}$ to $20.6 \pm 2.3 \mathrm{~mm}$ on the right. This represented a statistically significant increase of $11 \%$ on the left and and $12 \%$ on the right $(\mathrm{P}<0.05)$. Mean L5-S1 neuroforaminal height increased from $15.7 \pm 3.0 \mathrm{~mm}$ to $18.4 \pm 2.8 \mathrm{~mm}$ on the left and from $15.6 \pm 2.1 \mathrm{~mm}$ to $18.3 \pm 1.8 \mathrm{~mm}$ on the right. This represented a statistically significant increase of $17 \%$ on both sides at L5-S1 $(\mathrm{P}<0.05)$. Figure 5 shows the increase in neuroforaminal height at L5-S1 as the disc space is distracted. The slope was significantly greater than zero with approximately $1 \mathrm{~mm}$ gain in neuroforaminal height per revolution 
of the driver. Technically it meant the neuroforamen distracted by $1 \mathrm{~mm}$ on average for every complete revolution of the screw driver. Maximum distraction occurred between 2-3.5 revolutions of the driver. On continued attempts at distraction the screw started backing out at the sacral end. In each cadaver at least 2 full revolutions of screwdriver were allowed before the screw started backing out. Two out of the 5 cadavers allowed 3 turns, while one cadaver allowed 3.5 turns of distraction. As the disc space reached the point of maximum distraction the resistance to further distraction was found to increase exponentially. On continued attempts at distraction a clear give way was felt as screw started backing out at the sacral end. In the youngest lumbar specimen (which was 28 years of age), it was impossible to rotate the screw any further beyond maximum distraction. In addition, the screw did not back out on attempts at further distraction. There were no significant differences between the left and right neuroforaminal height measurements.

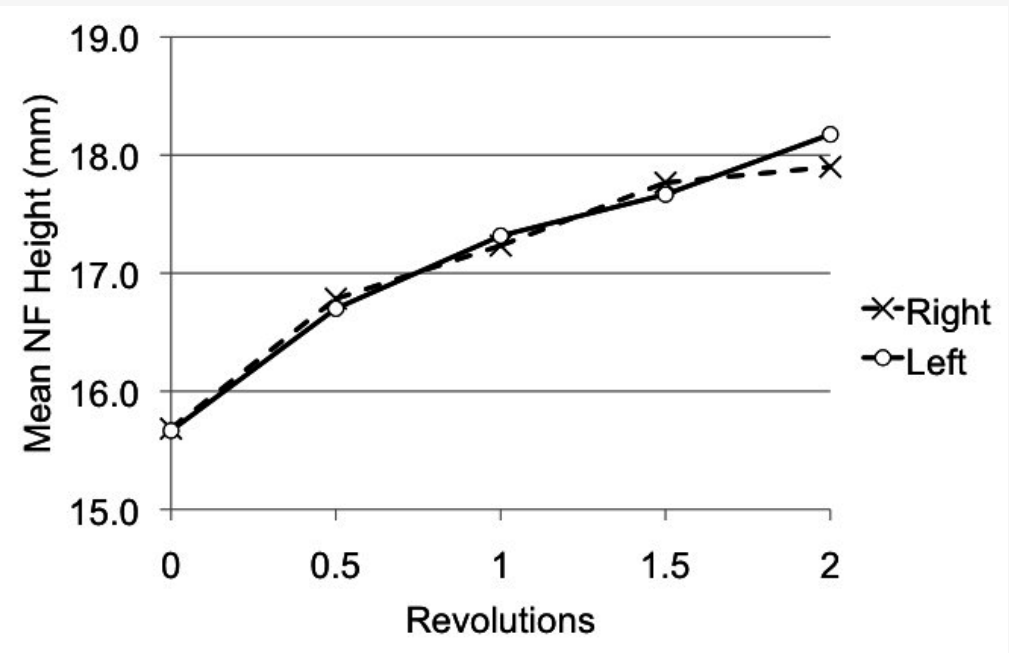

Fig. 5. The change in mean neuroforaminal height at L5-S1 based on the number of revolutions (turns) of the distraction rod. Changes in neuroforaminal height are similar between the left and right side and are approximately $1 \mathrm{~mm} /$ revolution.

\section{Discussion}

Degenerative disc disease in the lower lumbar spine can lead to stenosis of the lumbar spinal canal as well as the neuroforamen. In the lumbar spine the neuroforamen is shaped like an inverted tear drop. It is bounded superiorly and inferiorly by the pedicle of the adjacent vertebrae. The posterior boundary is formed by the pars interarticularis and ligamentum flavum. The anterior boundary is formed by the posteroinferior margin of the superior vertebral body, the posterior margin of the intervertebral disc, and the posterosuperior margin of the inferior vertebral body. ${ }^{34}$ Neuroforaminal height in the lumbar spine has been variously documented to range form $17 \mathrm{~mm}$ to $21 \mathrm{~mm}$. ${ }^{2,5}$ Degenerative changes in the motion segment can lead to loss of disc height and facet joint arthritis. This can lead to neuroforaminal stenosis caused by subluxation of the superior articular facet, protruding annulus and osteophytes from the posterolateral portion of the vertebral body. The dorsal root ganglion normally occupies approximately $23 \%$ to $30 \%$ of the area of the foramen and lies within the superior lateral portion of the lumbar 
intervertebral foramen directly below the pedicle in $90 \%$ of lumbar levels. ${ }^{6,7}$

Neuroforaminal stenosis caused by degenerative changes can lead to nerve root compression in the superoinferior or anteroposterior direction leading to symptomatic radiculopathy. Conventional fusion procedures can involve direct decompression of the neuroforamen by posterior approach to the lumbar spine. Alternatively the neuroforaminal size can be increased indirectly by anterior interbody distraction with spacers or posterior distraction with pedicle screws. ${ }^{7,8,9,10}$ Chen et al reported an increase in the neuroforaminal area by $29 \%$ atL4-L5 and by $33.8 \%$ at L5-S1 after ALIF using a cylindrical cage in a human cadaveric model. ${ }^{9}$ Wang et al reported a $10 \%$ increase in the foraminal height at L3-L4 after ALIF in fresh calf spines. Such indirect decompression of the nerve roots can relive radicular symptoms of stenosis. ${ }^{11}$ As opposed to direct decompression of the neuroforamen this reduces the handling of the nerve root and may prevent iatrogenic nerve root injury.

Transsacral instrumentation of lower lumbar spine is a novel approach to L4-S1 anterior interbody fusion. Accessing these disc spaces through the presacral approach when coupled with percutaneous posterior segmental fixation can reduce surgical time, trauma and post surgical recuperation period. The 2 level transsacral screw has been designed to distract the disc spaces and consequently the neuroforamina. At L4-L5 it can cause disc space distraction due to thread pitch differential between the L4 and L5 segments of the L4-L5 rod. At L5-S1 it allows manual control of disc space distraction using a specialized screw driver that engages a distraction rod that lags the L4-L5 rod and the sacral anchor of the screw as has been explained earlier (Figure 3). This cadaveric study was planned to evaluate the ability of this system to indirectly increase the neuroforaminal height. We found a significant increase in the neuroforaminal height at both L4-L5 and L5-S1 levels. At L4-L5 the foraminal height increased by $11 \%$ on the left and $12 \%$ on the right. At L5-S1 the foraminal height increased by $17 \%$ on both sides at the point of maximum distraction. Manual distraction mechanism at L5-S1 allowed on an average $1 \mathrm{~mm}$ distraction of the neuroforamen per turn of the screw driver. Maximum distraction allowed in cadavers varied, most likely based on bone quality and soft tissue pliability. All cadavers allowed at least 2 full turns of the screw driver. Two cadavers allowed 3 full turns while one cadaver allowed 3.5 turns. As the disc space got progressively distracted, further rotation of the screw driver became increasingly difficult signaling to the surgeon that the motion segment is approaching the point of maximum distraction. A clear give away followed by backing out of the screw at the sacral end was seen on continued attempts at distraction after maximum distraction was achieved. Thus in this cadaveric study this system of transsacral instrumentation was found to allow indirect increase in neuroforaminal size following disc space distraction. However it was found that manual distraction system for L5-S1 needs to be carefully used as maximum distraction allowed at L5-S1 is variable. Although it may not be possible to extrapolate these results as is to conditions in vivo, it is possible that most L5-S1 motion segments will allow 2 full turns of the screw driver or $2 \mathrm{~mm}$ of foraminal distraction. After this however if the surgeon wants to proceed with further distraction, careful consideration must be given to increasing resistance to distraction. Based on this cadaveric study distraction beyond 3 full turns of screw driver may risk screw back out. 
The results from this study have limitations. Firstly, in a clinical scenario it may more difficult to control the direction and placement of the screw as compared to that in a cadaver setting even with preoperative planning, use of fluoroscopy, and careful placement of guide wire in the desired direction and placement of the screw. Additionally and similarly to the majority of biomechanical studies examining fusion constructs, this data is representative of the immediate post-operative period and does not include the natural physiologic loading, stress relaxation, and biological remodeling that will occur clinically. Maintenance of neuroforaminal height will be dependent upon the subsidence during the fusion process, which is a complex process. In addition, the amount of disc space distraction is bound to be variable based on bone quality, soft tissue pliability, and loading conditions. Poor quality of sacral bone can lead to back out of the sacral anchor before desired distraction is achieved. It is possible that in some collapsed segments facet joint capsules and annulus may not be pliable enough to allow any significant distraction. The direction of the screw in the coronal plane, differential pliability of soft tissues on two sides as well as asymmetrical degeneration also may cause asymmetrical height gain. In tall discs the maximum tensile limit of the annulus and other soft tissues might be reached shortly after starting the distraction process. Also it might not be possible to rely entirely on indirect foraminal decompression using this system as a method of nerve root decompression in all cases.

Careful selection of cases, a detailed discussion with the patient regarding possibility of inadequate decompression and requirement of further surgical intervention will be prudent. Although limited conclusions can be drawn from this study it does help in refining the surgical technique for this novel approach and gives insight into the distraction process. Manual gradual controlled foraminal and disc space distraction at L5-S1 is allowed by the implant. It is suggested that surgeons should pay careful attention to the tactile feedback received during a slow and controlled distraction process. Although it is possible that most L5-S1 motion segments will allow at least 2 full turns of distraction screwdriver, it would be prudent to stop when there is an exponential increase in the resistance to distraction. This signals that the motion segment is reaching its distraction limit and should alert the surgeon to stop the distraction to prevent backing out of the sacral anchor. Before making any clear surgical recommendations, reliability and uses of this technology need to be explored further in a clinical setting with respect to the quantitative aspects of neuroforaminal distraction.

\section{References}

1. Erkan S, Wu C, Mehbod A, Hsu B, Pahl D, Transfeldt E (2009) Biomechanical evaluation of a new AxiaLIF technique for two-level lumbar fusion. Eur Spine J 18:807-14.

2. Cinotti G, De Santis P, Nofroni I, Postacchini F (20020 Stenosis of lumbar intervertebral foramen anatomic study on predisposing factors. Spine 27(3):223-9.

3. Vanderlinden RGF. (1984) Subarticular entrapment of the dorsal root ganglion as a cause of sciatic pain. Spine 9(1):19-22.

4. Crock HV. (1981) Normal and pathological anatomy of the lumbar spinal nerve root canals. J Bone Joint Surg [Br] 63:487-90. 
5. Fujiwara A, An HS, Lim T, Haughton VM. (2001) Morphologic changes in the lumbar intervertebral foramen due to flexion-extension, lateral bending, and axial rotation: An in vitro anatomic and biomechanical study. Spine 26(8):876-82.

6. Cohen MS, Wall EJ, Brown RA, Rydevik B, Garfin SR. (1990) Cauda equina anatomy II: Extrathecal nerve roots and dorsal root ganglia. Spine 15(12):1248-51.

7. Hasegawa T, Haughton VM, Nowicki BH. (1995) Critical heights of the intervertebral discs and foramina. A cryomicrotome study in cadavera. J Bone Joint Surg $[$ Am] 77(1):32-8.

8. Inufusa AA, An HS., Glover MJ, McGrady L, Tae-Hong L, Lee RH . (1996) The ideal amount of lumbar foraminal distraction for pedicle screw instrumentation. Spine 21(19):2218-23.

9. Chen DF, Fey LA, Lok J, Yuan P, Edwards TW, Yuan HA. (1995) Increasing neuroforaminal volume by anterior interbody distraction in degenerative lumbar spine. Spine 20(1):74-9.

10. Schlegel JD, Champine J, Taylor MS, Watson JT, Champine M, Schleusener RL, et al. (1994) The role of distraction in improving the space available in the lumbar stenotic canal and foramen. Spine 19(18):2041-7.

11. Wang M, Dalal S, Bagaria V, McGrady L, BS, Rao R. (2007) Changes in the lumbar foramen following anterior interbody fusion with tapered or cylindrical cages. The Spine Journal 7:563-9.

\section{Acknowledgement}

Only the instrumentation used in this study was provided by TranS1, Inc. (Wilmington, NC, U.S.A.). No research funds were received.

\section{Disclosures}

All authors declare that they have no conflict of interest with respect to this study.

\section{Corresponding Author}

Nathaniel Ordway, Orthopedic Surgery, SUNY Upstate University, ordwayn@upstate.edu.

Copyright (C) 2014 ISASS - International Society for the Advancement of Spine Surgery. To see more or order reprints or permissions, see http://ijssurgery.com. 